\title{
Sífilis gestacional: um estudo epidemiológico no Nordeste do Brasil
}

\author{
Gestational syphilis: an epidemiological study in Northeastern Brazil \\ Sífilis gestacional: un studio epidemiologico en el noreste de Brasil
}

Recebido: 11/05/2021 | Revisado: 18/05/2021 | Aceito: 22/05/2021 | Publicado: 08/06/2021

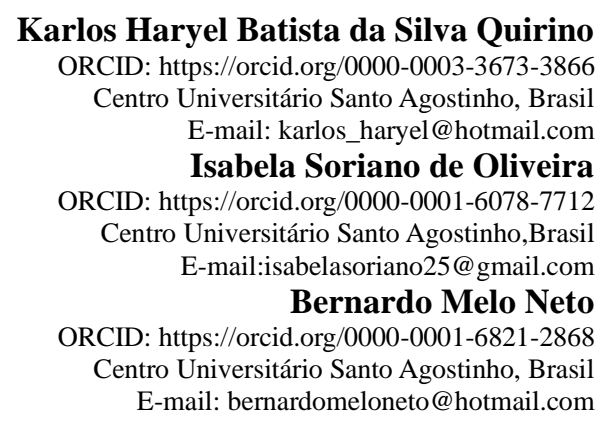

\begin{abstract}
Resumo
Esse estudo objetivou avaliar a incidência dos casos notificados de mulheres infectadas com sífilis durante a gestação e que residiam na região Nordeste do Brasil, no período de 2013-2020. Tratou-se de uma pesquisa de cunho epidemiológico, transversal e descritivo que empregou dados obtidos do Sistema de Informação de Agravo de Notificações (SINAN), disponibilizados na plataforma eletrônica do Departamento de Informática do Sistema Único de Saúde (DATASUS). Entre os anos de 2013 a 2020 foram notificados 63.908 casos de sífilis gestacional na região Nordeste do país, tendo o ano de 2018 com maior número de casos. Verificou-se que a sífilis apresentou maior incidência em mulheres gestantes jovens, entre 20-29 anos, de cor parda e de baixa escolaridade, o que mostrou que a doença acomete principalmente as populações mais vulneráveis, com reduzido acesso à saúde, educação e a informação. A menor incidência ocorreu em mulheres com idade acima de 40 anos, mulheres de raça/cor indígena e amarela, e as que possuem ensino superior completo. O Ministério da Saúde vem evoluindo no controle da sífilis gestacional, criando estratégias para fortalecer as redes de atenção à saúde, e esse empenho é percebido a partir do ano 2019, com a queda expressiva de notificações comparada aos anos anteriores. E esse esforço precisa ser mantido, com ajuda de toda população, para que não só a região Nordeste, mas todo o Brasil consiga controlar e diminuir cada vez mais a incidência dessa doença em mulheres gestantes.
\end{abstract}

Palavras-chave: Sífilis gestacional; Sistema de informação em saúde; Vigilância epidemiológica; Saúde pública.

\begin{abstract}
This study aimed to assess the incidence of reported cases of women infected with syphilis during pregnancy and who lived in the Northeast region of Brazil, in the period 2013-2020. It was an epidemiological, cross-sectional and descriptive research that used data obtained from the Notifiable Disclosure Information System (Sistema de Informação de Agravo de Notificação), available on the electronic platform of the Informatics Department of the Unified Health System (Departamento de Informática do Sistema Único de Saúde). Between 2013 and 2020, 63,908 cases of gestational syphilis were reported in the Northeast region of the country, with 2018 having the highest number of cases. It was found that syphilis had a higher number in young pregnant women, between 20-29 years old, brown in color and with low education, which revealed that the disease affects mainly the most vulnerable populations, with reduced access to health, education and information. The lower occurrence happened to women ager over 40, indigenous and yellow skin women and those with higher graduation degree. The Ministry of Health has been evolving in the control of gestational syphilis, creating strategies to strengthen health care networks, and this commitment is perceived from the year 2019, with the significant drop in notifications compared to previous years. And this effort needs to be maintained, with the help of the entire population, so that not only the Northeast region, but the whole of Brazil is able to control and increasingly reduce the incidence of this disease in pregnant women.
\end{abstract}

Keywords: Gestational syphilis; Healthy information system; Epidemiological surveillance; Public health.

\section{Resumen}

Este estudio tuvo como objetivo evaluar la incidencia de casos notificados de mujeres infectadas por sífilis durante el embarazo y que vivían en la región Nordeste de Brasil, en el período 2013-2020. Se trata de un estudio epidemiológico, transversal y descriptivo que utilizó datos obtenidos del Sistema de Información de Divulgación Notificable (SINAN), disponibles en la plataforma electrónica del Departamento de Informática del Sistema Único de 
Salud (DATASUS). Entre 2013 y 2020, se notificaron 63,908 casos de sífilis gestacional en la región Nordeste del país, siendo 2018 el mayor número de casos. Se encontró que la sífilis tuvo mayor incidencia en gestantes jóvenes, entre 20-29 años, de color café y con baja escolaridad, lo que evidenció que la enfermedad afecta principalmente a las poblaciones más vulnerables, con acceso reducido a salud, educación y información. La incidencia más baja ocurrió en mujeres mayores de 40 años, mujeres de raza/color indígena y amarilla, y aquellas que han completado la educación superior. El Ministerio de Salud ha ido evolucionando en el control de la sífilis gestacional, creando estrategias para fortalecer las redes de atención de la salud, y este compromiso se percibe desde el año 2019, con la caída significativa de las notificaciones en comparación con años anteriores. Y este esfuerzo debe mantenerse, con la ayuda de toda la población, para que no solo la región Nordeste, sino todo Brasil sea capaz de controlar y reducir cada vez más la incidencia de esta enfermedad en las mujeres embarazadas.

Palabras clave: Súfilis gestacional; Sistema de información de salud; Vigilancia epidemiológica; Salud pública.

\section{Introdução}

A sífilis é uma Infecção Sexualmente Transmissível (IST) conhecida desde o século XV, exclusiva do ser humano e causada pela bactéria Treponema pallidum. A principal via de transmissão é pelo contato sexual desprotegido, seguida pela transmissão vertical para o feto durante a gestação de uma mãe com sífilis não tratada ou tratada de forma inadequada, podendo também ser transmitida de forma indireta (contato com objetos contaminados) ou transfusão sanguínea (Brasil, 2010).

$\mathrm{Na}$ ausência de manifestações clínicas, o diagnóstico de sífilis é realizado por exames sorológicos. A triagem é feita por métodos conhecidos como não treponêmicos, por utilizarem antígenos não derivados do agente causal, sendo o teste do VDRL (Veneral Disease Research Laboratory) o mais realizado. A titulação é muito importante no controle da cura, através da comparação periódica dos títulos. Na fase primária o exame tem 70\% de sensibilidade, $99 \%$ na secundária, $75 \%$ na terciária, e na sífilis congênita também há uma alta especificidade de diagnóstico, chegando a 98\% (Saraceni, 2005).

Contrair a doença durante a gravidez pode causar aborto espontâneo, morte fetal ou neonatal, prematuridade e danos graves à saúde do concepto. Quando não é feito o tratamento da infecção materna, a chance de transmissão para o feto é de $80 \%$ a $100 \%$ dos casos. Mesmo após receber o tratamento, mulheres que contraíram sífilis durante a gestação, apresentam um risco maior de conseqüências adversas, comparado com mulheres sem histórico médico de infecção (Magalhães et al., 2011).

A cada ano, cerca de 2 milhões de gestantes são infectadas no mundo. A maior parte das gestantes não realiza o teste para sífilis, e as que o fazem não se tratam adequadamente ou sequer recebem tratamento. Alguns estudos associaram a doença a pobreza, infecção pelo HIV, abuso de drogas e subutilização do sistema de saúde, e demonstraram como fatores de risco individual as gestantes adolescentes, baixa escolaridade, história de infecções sexualmente transmissíveis (IST), raça/cor não branca, história de sífilis em gestações anteriores, múltiplos parceiros e baixa renda (Nonato et al., 2015).

Apesar de todo conhecimento da doença sobre seu agente etiológico, suas formas de transmissão e tratamento com altas taxas de cura, ainda se tem uma elevada incidência de casos de sífilis, e isso se deve a carência total de aplicação de protocolos, que caso fossem cumpridos, permitiriam a redução da disseminação da doença. No Brasil, mesmo a sífilis gestacional ser agravo de notificação compulsória desde 1986, a notificação só atinge 32\% dos casos, e esses dados refletem a precariedade nos serviços de assistência no período pré-natal e no parto (Campos et al., 2010; Damasceno et al., 2014).

O diagnóstico de sífilis feito no pré-natal junto com o tratamento imediato são cruciais para a quebra da cadeia de transmissão da doença ao feto. A droga utilizada e mais eficaz para tratar a sífilis é a penicilina benzatina, e a dose e tempo de tratamento variam de acordo com a fase da doença e do estado clínico da paciente, sendo que para afastar a possibilidade de infecção do concepto, o tratamento precisa ser finalizado pelo menos 30 dias antes do parto. A pesquisa por outras ISTs também faz parte do tratamento (Marques et al., 2018; Mascarenhas et al., 2016).

Nesse contexto, o objetivo desse estudo foi avaliar a incidência de casos de sífilis em gestantes na região nordeste do Brasil e os fatores que mais influem na ocorrência dessa doença nessa população, no período de 2013-2020. 


\section{Metodologia}

Esse estudo incluiu uma pesquisa de série temporal retrospectiva, de cunho epidemiológico, transversal com abordagem descritiva (Pereira et al., 2018). Os dados de notificação de casos de sífilis em gestantes foram coletados através do SINAN (Sistema de Informação de Agravos de Notificação) e divulgados na plataforma eletrônica DATASUS (Departamento de Informática do Sistema Único de Saúde), pertencente ao Ministério da Saúde. O estudo foi realizado nos meses de fevereiro e março de 2021, englobando os casos notificados de sífilis na região Nordeste do Brasil, entre os anos de 2013a 2020.

As variáveis avaliadas foram: faixa etária, cor ou raça, escolaridade, esquema de tratamento e classificação clínica. A análise dos dados se pôs de acordo com a estatística descritiva e os resultados foram apresentados em formas de tabelas e gráficos elaborados pelo software Microsoft Office Excel®.

\section{Resultados}

Verificou-se que na região nordeste do Brasil, a sífilis gestacional foi incidente em todos os anos, no período de 2013 a 2020. Neste intervalo de tempo foram notificados, no SINAN, 63.908 casos de mulheres acometidas pela sífilis durante a gravidez.

Figura 1. Número de casos de gestante com sífilis no Nordeste por ano de diagnóstico.

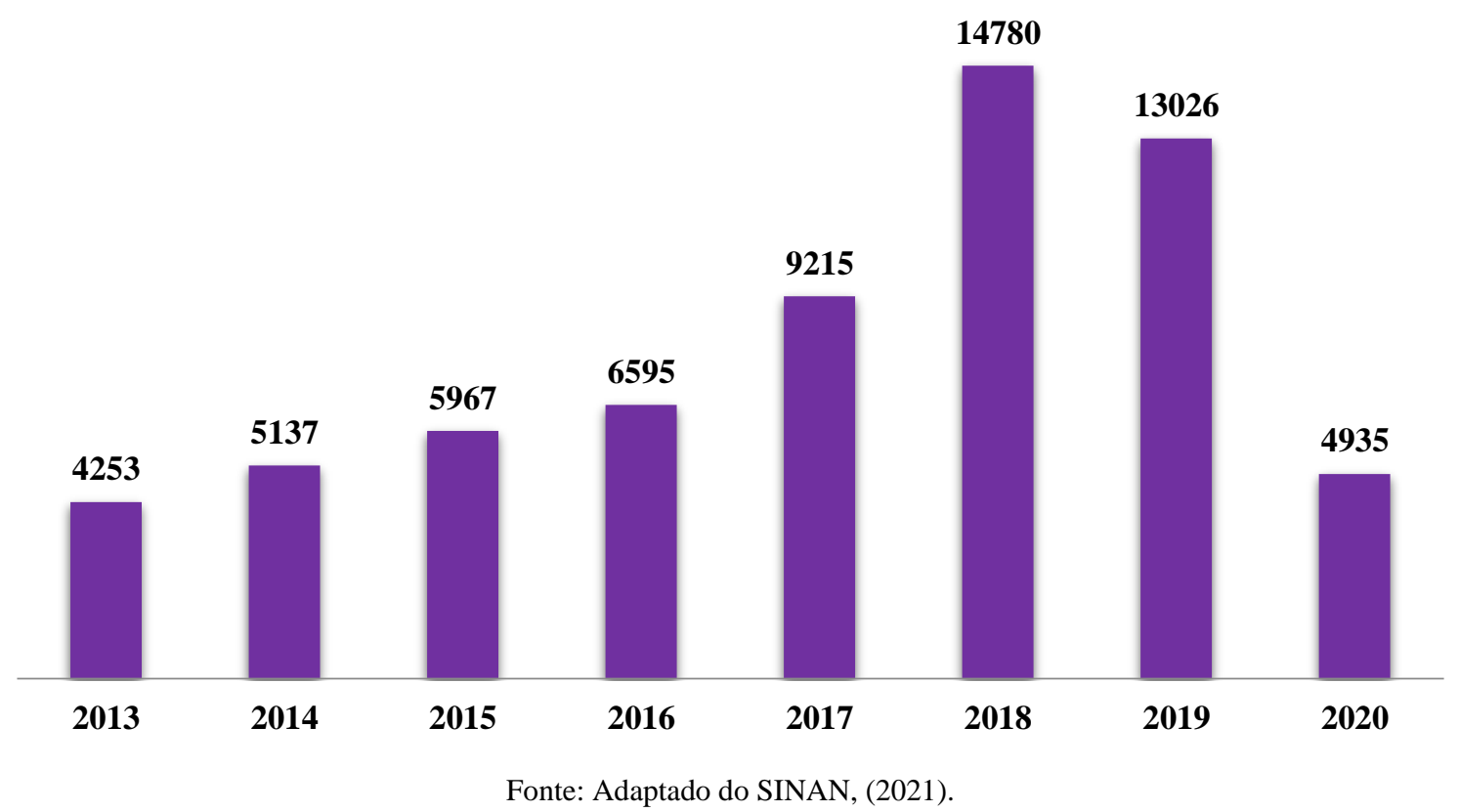

Constatou-se que entre os anos de 2013 e 2018 houve um aumento progressivo no número de casos de sífilis em gestantes no Nordeste, sendo 4.253 casos no ano de 2013 e 14.780 casos no ano de 2018. A partir do ano de 2019 houve uma queda no número de casos notificados, totalizando 13.026. No ano de 2020 o número de infecções continuou a cair, sendo apenas 4.935 notificações. 
Figura 2. Média da distribuição percentual de casos de gestantes com sífilis no Nordestesegundo faixa etária entre os anos de 2013 a 2020.

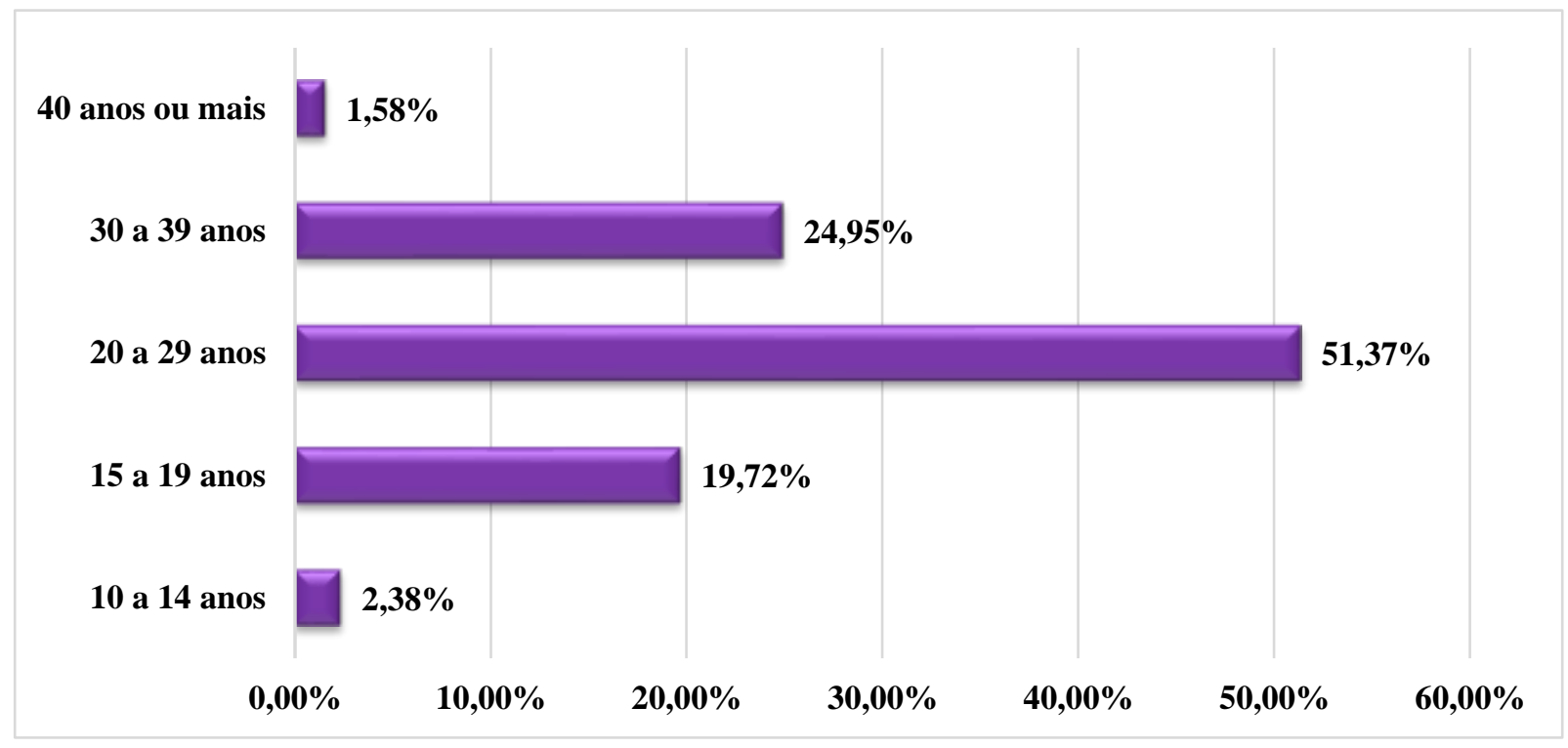

Fonte: Adaptado do SINAN, (2021).

A Figura 2 apresenta a média percentual de casos segundo a faixa etária. Observa-se que a maior detecção ocorreu em mulheres de 20 a 29 anos, segundo o SINAN, 33.162 gestantes foram infectadas (51,37\%), e o ano de 2018 teve a maior detecção de casos. A faixa etária de 30 a 39 foi a segunda mais notificada, com 12.565 casos $(24,95 \%)$.

Figura 3: Média da distribuição percentual de casos de gestantes com sífilisno Nordeste segundo cor ou raça entre os anos de 2013 a 2020.

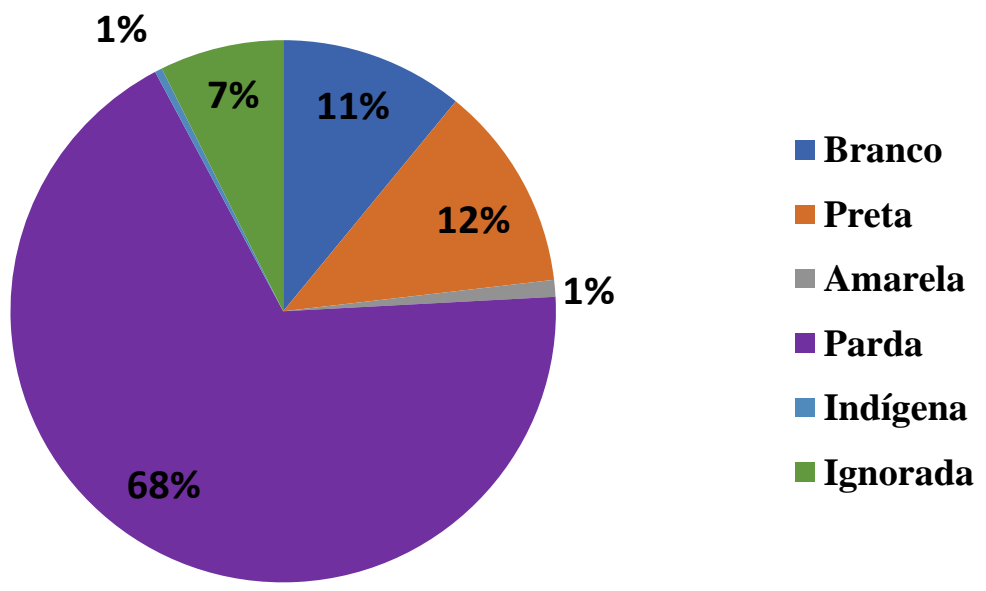

Fonte: Adaptado do SINAN, (2021).

A Figura 3 apresenta a média percentual de casos de gestantes que contraíram sífilis de acordo com a cor ou raça. 
Observa-se que a cor parda foi a mais notificada, com 43.724 notificados (68\%). A cor preta vem logo em seguida com segundo maior número de registros, 7.678 (12\%).

Tabela 1. Casos de gestantes com sífilis segundo escolaridade por ano de diagnóstico.

\begin{tabular}{|l|l|l|l|l|l|l|l|l|l|}
\hline Escolaridade & $\mathbf{2 0 1 3}$ & $\mathbf{2 0 1 4}$ & $\mathbf{2 0 1 5}$ & $\mathbf{2 0 1 6}$ & $\mathbf{2 0 1 7}$ & $\mathbf{2 0 1 8}$ & $\mathbf{2 0 1 9}$ & $\mathbf{2 0 2 0}$ \\
\hline Analfabeto & 76 & 109 & 95 & 90 & 103 & 132 & 99 & 33 \\
\hline $\mathbf{1}^{\mathbf{a}}$ a 4 & $\mathbf{a}$ série incompleta & 438 & 524 & 536 & 538 & 665 & 946 & 752 & 293 \\
\hline $\mathbf{4}^{\mathbf{a}}$ série completa & 249 & 271 & 303 & 364 & 427 & 601 & 544 & 206 \\
\hline $\mathbf{5}^{\mathbf{a}}$ a $\mathbf{8}^{\mathrm{a}}$ série incompleta & 881 & 1.133 & 1.355 & 1.427 & 2.060 & 3.448 & 2.981 & 1.045 \\
\hline Fundamental completo & 269 & 304 & 476 & 553 & 778 & 1.230 & 1.129 & 440 \\
\hline Médio Incompleto & 426 & 504 & 649 & 736 & 1.147 & 1.834 & 1.704 & 611 \\
\hline Médio Completo & 469 & 654 & 714 & 833 & 1.435 & 2.604 & 2.427 & 978 \\
\hline Superior Incompleto & 16 & 30 & 51 & 45 & 90 & 129 & 126 & 63 \\
\hline Superior Completo & 18 & 28 & 28 & 42 & 67 & 136 & 118 & 43 \\
\hline Não se aplica & & $2-$ & & 3 & 2 & 2 & $1-$ & \\
\hline Ignorado & 1.411 & 1.578 & 1.760 & 1.964 & 2.441 & 3.718 & 3.145 & 1.223 \\
\hline
\end{tabular}

Fonte: Adaptado do SINAN, (2021).

A Tabela 1 evidencia os casos de sífilis em gestantes de acordo com a escolaridade, tendo uma grande proporção de mulheres que cursaram da $5^{\mathrm{a}}$ à $8^{\mathrm{a}}$ série do ensino fundamental, com 14.330 casos no período estudado, seguido de mulheres que possuem apenas o ensino médio completo, com 10.114 casos.

Com relação ao esquema de tratamento, o DATASUS disponibilizou apenas os dados referentes ao período de 2016 a 2019. A Tabela 2 mostra que a Penicilina foi a forma de tratamento mais utilizada pelas gestantes.

Tabela 2. Distribuição percentual de casos de gestantes com sífilis segundo esquema de tratamento por ano de diagnóstico.

\begin{tabular}{|c|c|c|c|c|}
\hline Esquema de Tratamento & 2016 & 2017 & 2018 & 2019 \\
\hline Penicilina & $86,8 \%$ & $87,2 \%$ & $86,7 \%$ & $87 \%$ \\
\hline Outro esquema & $2,5 \%$ & $4 \%$ & $2,5 \%$ & $2 \%$ \\
\hline Não realizado & $4,4 \%$ & $4,2 \%$ & $6,4 \%$ & $6,5 \%$ \\
\hline Ignorado & $6,4 \%$ & $4,5 \%$ & $4,4 \%$ & $4,4 \%$ \\
\hline
\end{tabular}

Fonte: Adaptado do SINAN, (2021).

Os dados da Figura 4 demonstram que a maior parte das gestantes notificadas tiveram conhecimento da doença já no estágio inicial ou primário. Outra grande parte só tiveram o diagnóstico da infecção, com a doença em estado mais avançado, ou seja, em estado latente. 
Figura 4. Quantitativo de casos notificados de gestantes com sífilis segundo classificação clínica (2013-2020).

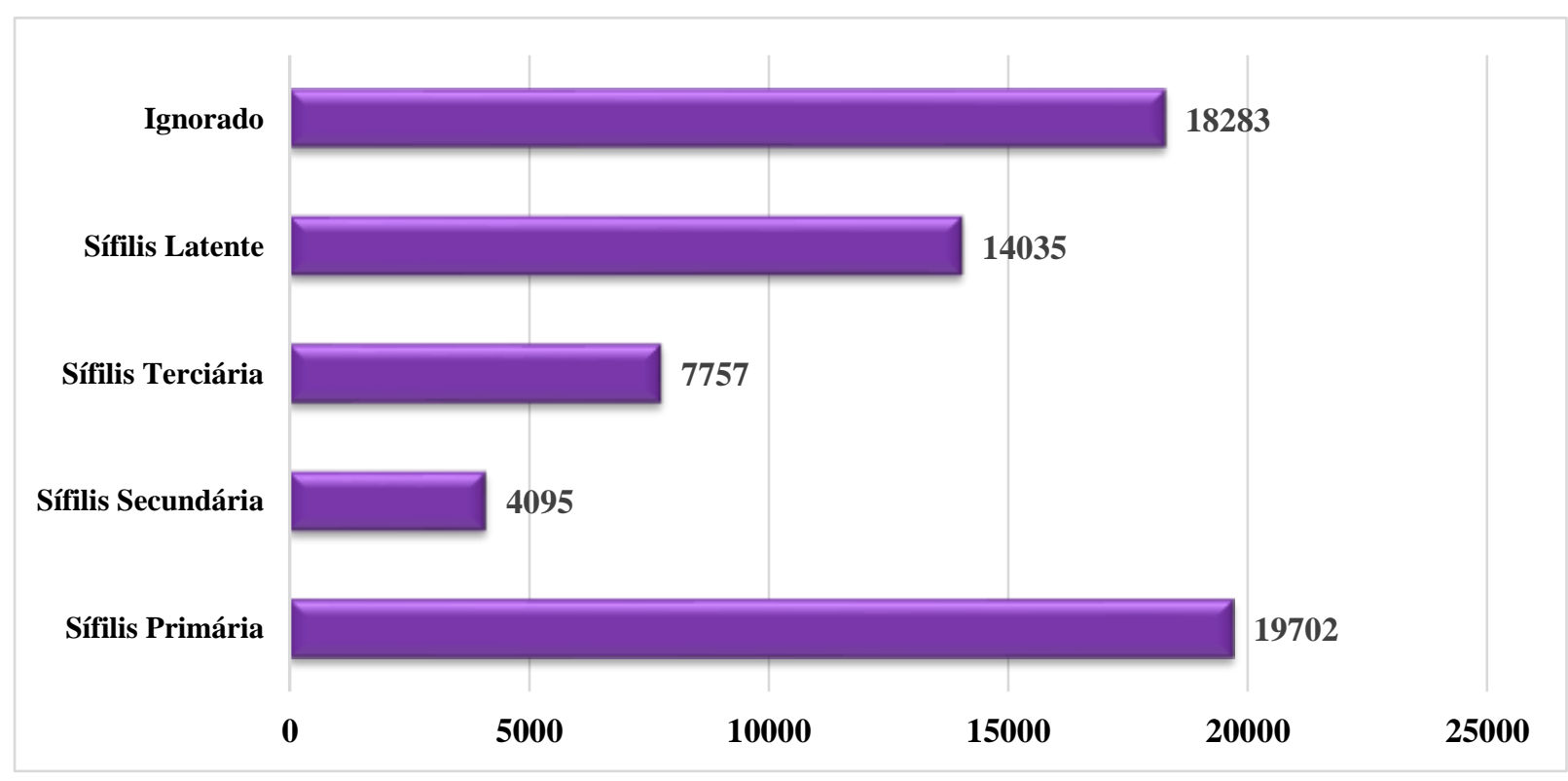

Fonte: Adaptado do SINAN, (2021).

\section{Discussão}

O quantitativo de casos de sífilis em gestantes no Nordeste do Brasil, apresentado na Figura 1, evidenciou uma elevação de casos entre os anos 2013 a 2018. Em um estudo realizado por Marques e colaboradores (2018), uma das hipóteses para que se tenha um aumento no número de casos é a ampliação no uso de testes rápidos em gestantes e a uma mudança comportamental, que pode ser a diminuição do uso de métodos contraceptivos (Marques, et al., 2018).

Em 2019, foi registrada uma queda no número de casos de sífilis gestacional, com relação ao ano anterior. No Brasil, o número total de casos notificados foi 3,3\% menor que o ano anterior, e isso pôde refletir na região Nordeste. Para que isso pudesse ocorrer o Ministério da Saúde realizou e vem implementando diversas estratégias nacionais para o controle da doença no Brasil, que incluem o fortalecimento das redes de atenção à saúde e o sistema de vigilância para enfretamento à sífilis (Brasil, 2020).

De acordo com a Figura 2, durante o período estudado, prevaleceu o diagnóstico de sífilis gestacional em mulheres jovens, com idade entre 20 a 29 anos. Um estudo feito por Lima e colaboradores (2017), na cidade de Sobral-Ceará, também identificou uma predominância na faixa etária de 20 a 25 anos, seguida da faixa etária de 26 a 30 anos (Lima, et al., 2017).

Em todo Nordeste, as cores de pele ou raça mais presente entre as mulheres grávidas infectadas por sífilis, foi a parda. Porém, isso não significa que a doença tenha acometimento sob determinada raça/cor, pois a maioria da população brasileira se autodeclara parda, devido à miscigenação (Moura, et al.,2019).

Em um estudo feito por Leal e colaboradores (2017), ele afirma que em comparação às mulheres brancas, mulheres pardas e pretas se concentram mais nas regiões Nordeste e Norte, como também demonstra que estas sofrem mais desvantagens e desigualdades em relação à assistência à saúde, como não receber um pré-natal adequado. Isso pode ter contribuído na grande presença de mulheres pardas acometidas por sífilis durante a gestação (Leal, et al., 2017).

No que diz respeito à escolaridade, o maior percentual de casos ocorreu em mulheres que não tinham o ensino fundamental completo, com grau de escolaridade entre a $5^{\circ}$ e a $8^{a}$ série. O estudo realizado mostrou um total de 14.330 
mulheres nessa categoria (Tabela 1). Essa distribuição é corroborada por um estudo realizado por Domingues e colaboradores (2014), que apontou que mulheres de menores escolaridades tiveram as coberturas mais baixas de testagem e consequentemente apresentaram maior prevalência de sífilis na gestação (Domingues, et al., 2014).

Raça ou cor não branca, baixo nível de escolaridade, como também ausência de um trabalho remunerado são variáveis que mais se associam estatisticamente à sífilis gestacional. Geralmente, são indivíduos que possuem uma condição socioeconômica menos favorecida e menor chance de acesso a um serviço de saúde de qualidade. Apesar dessas populações mais vulneráveis serem as mais acometidas com a sífilis, não se pode condicionar essa doença somente a elas, pois qualquer pessoa, independente da condição social ou econômica pode adquirir a infecção (Padovani, et al., 2018).

No que diz ao tratamento, a penicilina benzatina foi a droga mais utilizada durante o estudo realizado, e essa preferência se dar pelo fato da penicilina ser capaz de atravessar a barreira transplacentária e tratar mãe e feto ao mesmo tempo. O esquema constitui de uma dose padrão de 2,4 milhões UI, de forma intramuscular (IM), aplicado nos dois glúteos. A quantidade de doses varia de acordo com a condição clínica da paciente (Tabela 2) (Moreira, et al., 2017).

A ceftriaxona é outra droga aventada como tratamento alternativo em caso de indisponibilidade ou alergia ao tratamento penicilinico. Esse antibiótico, que faz parte do grupo das cefalosporinas, é bem tolerado pelo organismo, e se mostra uma alternativa interessante quando utilizado durante a sífilis primária e secundária. Ainda não existe um consenso com relação a sua posologia, e no caso da sífilis gestacional a forma mais utilizada é1.000mg via IM ou endovenosa, sendo sua aplicação diária por até 10 dias (Coelho \& Coelho, 2018).

O Ministério da Saúde informa que é difícil o diagnóstico clínico de sífilis em gestantes, principalmente na fase primária, isso devido o cancro duro além de ter um tempo curto e limitado de permanência, não causa outros sintomas e geralmente está localizado em áreas de difícil visualização: parede vaginal, cérvix ou períneo. O presente estudo mostrou um maior número de gestantes com classificação primária da doença (Figura 4), diferentemente do que se espera em um rastreamento, que a classificação latente predomina mais. Isso pode ter sido causado por algum equívoco ou erro de classificação, o que pode levar a tratamentos inadequados, e ressalta-se que quando não é possível estabelecer uma condição clínica que a paciente se encontra, esta deve ser classificada como sífilis de duração ignorada, tendo o tratamento preconizado com três doses de penicilina benzatina (Cardoso, et al., 2018; Cavalcante, et al., 2017).

A sífilis é uma doença sexualmente transmissível, e seu diagnóstico durante a gestação desperta tensão tanto por questões biológicas, quanto por repercussões sociais. Sentimentos de ansiedade, tristeza e medo constituem um pontencializador das vulnerabilidades existentes, dificultando a adesão ao tratamento e acompanhamento, que precisa ser feito adequadamente, para que se diminua o risco de transmissão da doença da mãe para o bebê. Além disso, mesmo que a mãe seja tratada corretamente, o não tratamento do parceiro implica em alto risco de reinfecção da gestante, o que pode voltar no aumento do risco de transmissão vertical da doença. Portanto, o enfretamento da epidemia de sífilis demanda habilidades de comunicação, ações intersetoriais e de vigilância em saúde, com ética e a garantia de confiabilidade das informações de toda equipe profissional (Figueiredo, et al.,2020; Mascarenhas, et al.,2016).

Entre as limitações do presente estudo, está o quantitativo de variáveis que apresentaram campos ignorados, o que dificulta uma análise mais completa dos dados apresentados. Outra limitação desta pesquisa são as informações provenientes de fontes secundárias, uma vez que esses dados dependem diretamente da atualização do sistema, podendo haver falhas na alimentação e disponibilidade nos sistemas de notificação. 


\section{Considerações Finais}

Conforme exposto, foi possível identificar que a região Nordeste do Brasil apresentou uma tendência crescente relacionada ao número de casos de sífilis, durante os anos de 2013 a 2018, seguido de uma tendência decrescente a partir do ano de 2019. Isso pode ser justificado pelas ações realizadas pelo Ministério da Saúde para o controle da doença.

A Pesquisa constatou a predominância de sífilis gestacional em mulheres jovens entre 20 a 29 anos, com classificação clínica primária, e inclinação para grupos populacionais específicos, como mulheres pardas e negras e baixo nível de escolaridade, representantes de classes socioeconômicas menos favorecidas.

Contudo, é de grande importância o contínuo monitoramento das variáveis utilizadas nesse estudo, para que se possa avaliar o resultado das ações e políticas públicas direcionadas ao controle da sífilis gestacional e orientar as tomadas de decisões relacionadas à doença.

Para reduzir a prevalência de sífilis na gestação, é imprescindível que os profissionais de saúde e toda a comunidade atentem para importância do diagnóstico precoce e tratamento eficaz da doença. Destaca-se também a importância da notificação de casos junto aos órgãos estaduais e municipais responsáveis pelos estudos epidemiológicos, para que se tenha a compreensão da evolução da doença, como também a implantação de medidas de controle e erradicação nas áreas mais afetadas.

\section{Referências}

Campos, A. L. D. A., Araújo, M. A. L., Melo, S. P. D., \& Gonçalves, M. L. C. (2010). Epidemiologia da sífilis gestacional em Fortaleza, Ceará, Brasil: um agravo sem controle. Cadernos de Saúde Pública, 26(9), 1747-1755.

Cardoso, A. R. P., Araújo, M. A. L., Cavalcante, M. D. S., Frota, M. A., \& Melo, S. P. D. (2018). Análise dos casos de sífilis gestacional e congênita nos anos de 2008 a 2010 em Fortaleza, Ceará, Brasil. Ciência \& Saúde Coletiva, 23, 563-574.

Cavalcante, P. A. D. M., Pereira, R. B. D. L., \& Castro, J. G. D. (2017). Sífilis gestacional e congênita em Palmas, Tocantins, 20072014. Epidemiologia e Serviços de Saúde, 26, 255-264.

Coelho, L. F., \& Coelho, C. M. (2019). Tratamento De Sífilis Com Ceftriaxona E Sua Eficácia Na Prevenção Da Sífilis Congênita. Revista da Faculdade de Medicina de Teresópolis, 3(2).

Damasceno, A. B., Monteiro, D. L., Rodrigues, L. B., Barmpas, D. B. S., Cerqueira, L. R., \& Trajano, A. J. (2014). Sífilis na gravidez. Revista Hospital Universitário Pedro Ernesto, 13(3).

Domingues, R. M. S. M., Szwarcwald, C. L., Souza Junior, P. R. B., \& Leal, M. D. C. (2014). Prevalência de sífilis na gestação e testagem pré-natal: Estudo Nascer no Brasil. Revista de Saúde Pública, 48, 766-774.

Figueiredo, D. C. M. M. D., Figueiredo, A. M. D., Souza, T. K. B. D., Tavares, G., \& Vianna, R. P. D. T. (2020). Relação entre oferta de diagnóstico e tratamento da sífilis na atenção básica sobre a incidência de sífilis gestacional e congênita. Cadernos de Saúde Pública, 36, $\mathrm{e} 00074519$.

Leal, M. D. C., Gama, S. G. N. D., Pereira, A. P. E., Pacheco, V. E., Carmo, C. N. D., \& Santos, R. V. (2017). A cor da dor: iniquidades raciais na atenção pré-natal e ao parto no Brasil. Cadernos de Saúde Pública, 33, e00078816.

Lima, V. C., Mororó, R. M., Martins, M. A., Ribeiro, S. M., \& Linhares, M. S. C. (2017). Perfil epidemiológico dos casos de sífilis congênita em um município de médio porte no nordeste brasileiro. Journalof Health \&BiologicalSciences, 5(1), 56-61.

Magalhães, D. M. D. S., Kawaguchi, I. A. L., Dias, A., \& Paranhos Calderon, I. D. M. (2011). A sífilis na gestação e sua influência na morbimortalidade materno-infantil. Comun. ciênc. saúde, 43-54.

Marques, J. V. S., Alves, B. M., Marques, M. V. S., Arcanjo, F. P. N., Parente, C. C., \& Vasconcelos, R. L. (2018). Perfil epidemiológico da sífilis gestacional: clínica e evolução de 2012 a 2017. SANARE-Revista de Políticas Públicas, 17(2).

Mascarenhas, L. E. F., Araújo, M. D. S. S., \&Gramacho, R. D. C. C. V. (2016). Desafios no tratamento da sífilis gestacional.

Ministério da Saúde (2010). Sífilis: Estratégias para Diagnóstico no Brasil. Brasília: Ministério da Saúde, Coordenação de Doenças Sexualmente Transmissíveis e Aids. 2010. 100 p. (Série TELELAB). 
Research, Society and Development, v. 10, n. 6, e51210616001, 2021

(CC BY 4.0) | ISSN 2525-3409 | DOI: http://dx.doi.org/10.33448/rsd-v10i6.16001

Ministério da Saúde (2020). Boletim Epidemiológico Sífilis 2020. Secretaria de Vigilância em Saúde. Ministério da Saúde. Número Especial. Out. 2020.

Ministério da Saúde, (2020). Brasil avança no enfretamento à sífilis. http://www.aids.gov.br/pt-br/noticias/brasil-avanca-no-enfrentamentosifilis\#: :text=DADOS\%20DE\%20S\%C3\%8DFILIS\%20NO\%20BRASIL\&text=Houve\%20redu\%C3\%A7\%C3\%A3o\%20de\%204\%2C5,an o\%20anterior\%20(63.182\%20 casos)

Moreira, K. F. A., de Oliveira, D. M., de Alencar, L. N., Cavalcante, D. F. B., de Sousa Pinheiro, A., \& Orfão, N. H. (2017). Perfil dos casos notificados de sífilis congênita. Cogitare Enfermagem, 22(2).

Moura, M. C. L. (2019). Sífilis congênita no Piauí: Um agravo sem controle. BrazilianJournalofSurgeryandClinicalResearch-BJSCR, 26(3), 29-35.

Nonato, S. M., Melo, A. P. S., \& Guimarães, M. D. C. (2015). Sífilis na gestação e fatores associados à sífilis congênita em Belo HorizonteMG, 2010-2013. Epidemiologia e Serviços de Saúde, 24, 681-694.

Padovani, C., de Oliveira, R. R., \& Pelloso, S. M. (2018). Sífilis enlagestación: asociación de las características maternas y perinatalesen una regióndelsur de Brasil1. Rev. Latino-Am. Enfermagem, 26, e3019.

Pereira, A. S., Shitsuka, D. M., Parreira, F. J., Shitsuka, R. (2018). Metodologia da pesquisa científica. [e-book]. Santa Maria, RS: UFSM/NTE. https://repositório.ufsm.br/bitstream/handle/1/15824/Lic_Computacao_Metodologia-Pesquisa-Científica.pdf?sequence=1 .

Saraceni, V. (2005). A sífilis, a gravidez e a sífilis congênita. Rio DST/AIDS, 1-22. 